\title{
Psychosis: An Emerging Field for EMDR Research and Therapy
}

\author{
Paul de Bont (1) \\ Mental Health Organization (MHO) GGZ Oost Brabant Land van Cuijk en Noord Limburg, Boxmeer, the Netherlands \\ Radboud University Nijmegen, NijCare, the Netherlands \\ Ad de Jongh \\ University of Amsterdam and VU University Amsterdam, Amsterdam, the Netherlands \\ PSYTREC, Bilthoven, the Netherlands \\ University of Worcester, Worcester, United Kingdom \\ Queen's University, Belfast, Northern Ireland \\ David van den Berg \\ Parnassia Psychiatric Institute, Den Haag, the Netherlands \\ VU University, Amsterdam, the Netherlands
}

\begin{abstract}
It has only been in this last decade that trauma-focused treatments (TFT) have been studied in patients with psychotic disorders. Before, the paradigm stated that TFT was contraindicated in these patients because clinicians and researchers assumed the risk of exacerbation of symptoms was too high. The purpose of this article is to examine the effectiveness of eye movement desensitization and reprocessing (EMDR) therapy in the treatment of psychosis. To this end, we will present a brief narrative review of the current state of research in this particular field. The results suggest that, contrary to the "no-TFTin-psychosis" paradigm, TFTs such as EMDR therapy can successfully be used to reduce trauma-related symptoms in patients with psychosis. Moreover, there are now provisional indications that psychotic symptoms such as delusions and hallucinations can be targeted directly and indirectly using EMDR therapy.
\end{abstract}

Keywords: psychosis; eye movement desensitization and reprocessing (EMDR) therapy; review; schizophrenia spectrum disorders; trauma

$\mathbf{T}$ here is a great need for effective trauma-focused treatments (TFT), such as eye movement desensitization and reprocessing (EMDR) therapy, in individuals with psychosis. As a group, patients with psychotic disorders more often report having been exposed to traumatic events in their lives than individuals in the general population (Matheson, Shepherd, Pinchbeck, Laurens, \& Carr, 2013; Varese et al., 2012). Also, there is a large body of evidence suggesting that exposure to traumatic events, especially during childhood, contributes to the occurrence of psychotic experiences (Bendall, Alvarez-Jimenez, Hulbert, McGorry, \& Jackson, 2012; Bentall, Wickham, Shevlin, \& Varese, 2012; Björkenstam,
Burström, Vinnerljung, \& Kosidou, 2016; Trotta, Murray, \& Fisher, 2015; Varese et al., 2012). Most studies show a dose-response relationship; that is, the higher the level of trauma exposure, the greater the risk of developing psychosis (Shevlin, Houston, Dorahy, \& Adamson, 2007; Varese et al., 2012). Evidence suggests that the first trauma-related onset of psychotic symptoms may occur in childhood (Arseneault et al., 2011). In addition to the finding that childhood adversities increase the risk of a future onset of psychosis, it has been found that traumas in adulthood often (immediately) precede the onset of psychosis (Linscott \& Van Os, 2013). An important research finding is that once an individual is suffering 
from psychosis, he or she is at an increased risk of exposure to new traumatic events, and the person may develop posttraumatic stress disorder (PTSD) as a consequence of experiencing an episode of psychosis (Maniglio, 2009; Mueser, Lu, Rosenberg, \& Wolfe, 2010; Rodrigues \& Anderson, 2017).

One of the by-effects of having a history of (childhood) trauma in people with psychosis is that it appears to worsen treatment adherence and treatment response to antipsychotic medication (Hassan \& De Luca, 2015; Lecomte et al., 2008). In addition, there is evidence suggesting that people with both a schizophrenia spectrum disorder and PTSD show an elevated risk of revictimization (Kuijpers, van der Knaap, \& Winkel, 2012; Maniglio, 2009). Moreover, the joint impact of psychosis and PTSD seems to increase positive psychotic symptoms, general psychopathology, and neurocognitive impairment, and to decrease general functioning and quality of life (Lysaker \& LaRocco, 2008; Lysaker, Buck, \& LaRocco, 2007; Sautter et al., 1999; Seow et al., 2016).

The main purpose of this article is to examine the effectiveness of EMDR therapy in the treatment armamentarium for individuals with psychosis. Firstly, we focus on the assessment of trauma and PTSD in patients with psychosis. Next, we discuss the pros and potential cons of TFT in patients with psychosis. Thirdly, we will examine the evidence concerning the role of EMDR therapy in the treatment of psychotic symptoms. Finally, we discuss some new initiatives in clinical practice and research in the area of trauma-focused therapy and psychosis and draw some general conclusions about the importance and the place of EMDR therapy in the treatment of psychosis.

\section{Assessment of Trauma and PTSD in Patients With Psychosis}

The importance of assessing trauma and PTSD in patients with psychotic disorders cannot be emphasized strongly enough and is in fact part of the guidelines of the National Institute for Health and Care Excellence (NICE, 2014). Although awareness of this fact is increasing in clinical practice, the available data suggest that both trauma exposure and PTSD remain undetected in most patients with psychotic disorders (De Bont et al., 2015; Lommen \& Restifo, 2009). This omission can be solved by implementing routine screening questionnaires such as the adapted and validated Trauma Screening Questionnaire (TSQ) for preselecting individuals with psychosis who report PTSD symptoms and subsequently offering them diagnostic clinical interviews to diagnose PTSD (de Bont et al., 2015). Clearly, without detecting the individuals concerned it is not very likely that TFT will be considered or applied at all.

\section{The Effectiveness of EMDR Therapy for Patients With Psychosis}

Treating trauma can be distressing and challenging. As a result, many researchers and clinicians assume that TFT can be harmful for patients with psychosis, resulting in exclusion of this patient group from TFT (Meyer, Farrell, Kemp, Blakey, \& Deacon, 2014; Ronconi, Shiner, \& Watts, 2014). The existing empirical evidence, however, does not appear to support the need for exclusion of psychotic patients for TFT. Table 1 presents an overview of studies using EMDR in patients with psychotic symptomatology. Studies were found using PubMed (searches related to "trauma," "PTSD," "psychosis," "EMDR," "traumafocused treatment").

\section{EMDR for PTSD in Patients With Psychosis}

Explorative studies examining TFT for comorbid PTSD in patients with psychotic disorders found positive effects on PTSD symptoms and little (WardBrown et al., 2018) to no detrimental side effects; in addition, research found that applying TFT for PTSD yielded neutral to positive side effects on psychosis, depression, coping, or quality of life, when using EMDR therapy (De Bont, Van Minnen, \& De Jongh, 2013; Laugharne et al., 2014; Van den Berg \& Van der Gaag, 2012; Ward-Brown et al., 2018) and traumafocused cognitive behavioral therapy (CBT; De Bont et al., 2013; Frueh et al., 2009; Mueser et al., 2015; Mueser et al., 2008; Ward-Brown et al., 2018).

The results of de Bont et al.'s (2013) hallmark research project in patients with psychosis and PTSD showed that augmenting care as usual with EMDR or prolonged exposure (PE) therapy for PTSD significantly reduced PTSD (van den Berg et al., 2015), results that were maintained up to the 12 -month follow-up (Van den Berg et al., 2018). The same held true for patients in the sample who fulfilled the criteria of the dissociative subtype of PTSD (Van Minnen et al., 2016). Secondarily, remission from psychosis was fostered, the severity of delusional/paranoid thinking in EMDR and PE therapy was reduced, and depression in PE appeared to diminish, but effects on auditory hallucinations and social functioning were found to stay neutral (De Bont et al., 2016; Van den Berg et al., 2018). Furthermore, compared to treatment as usual 


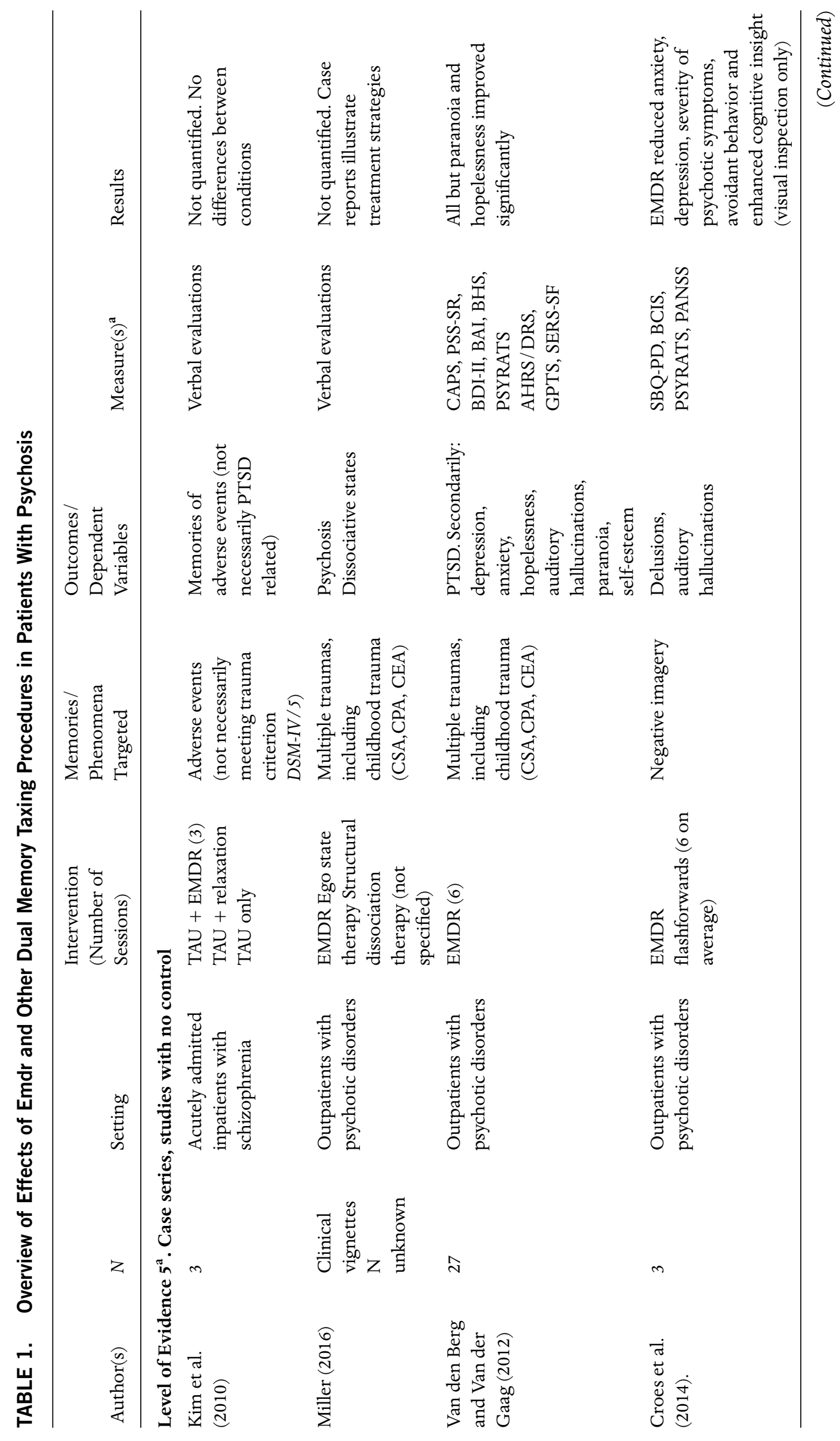




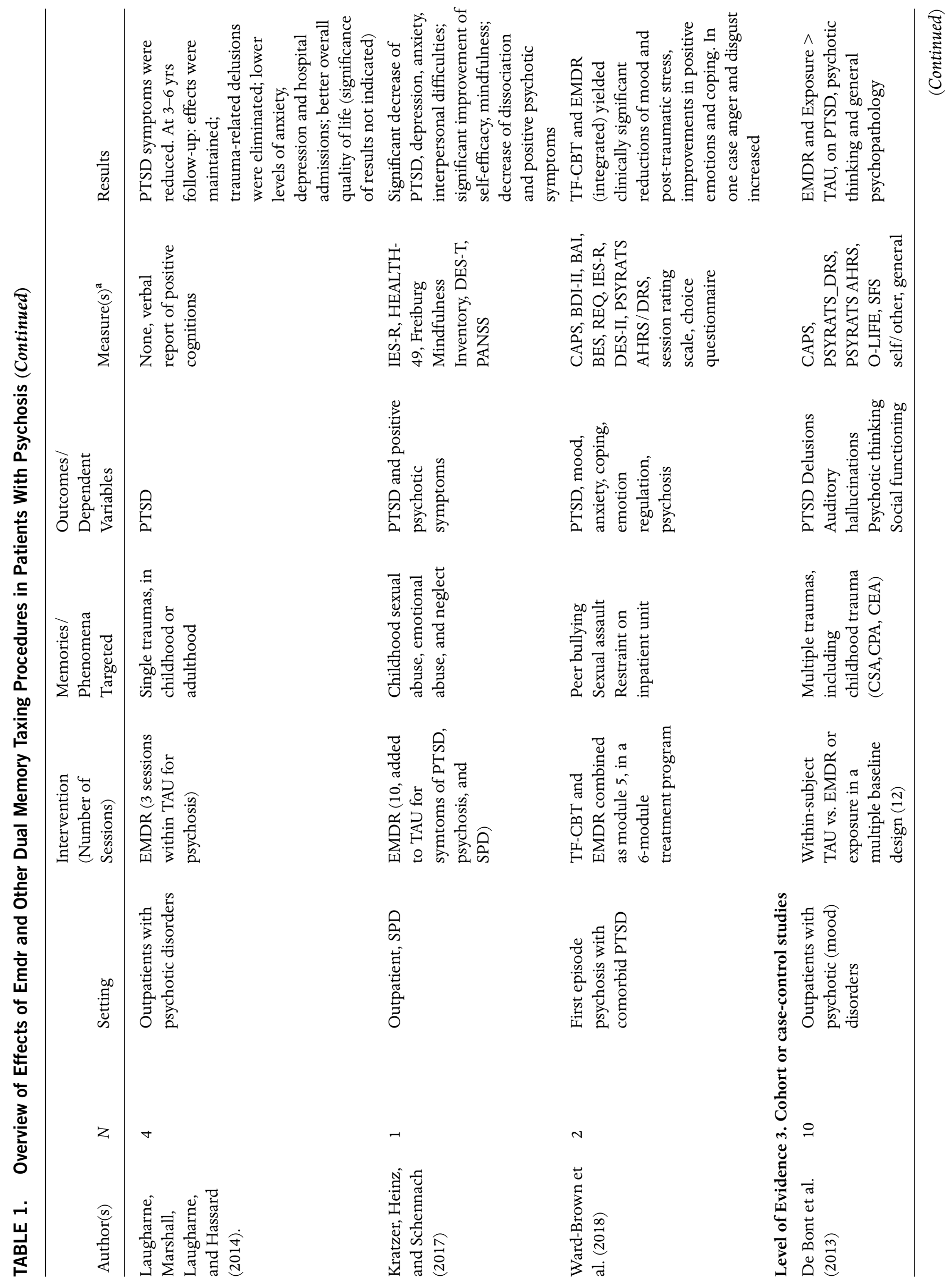




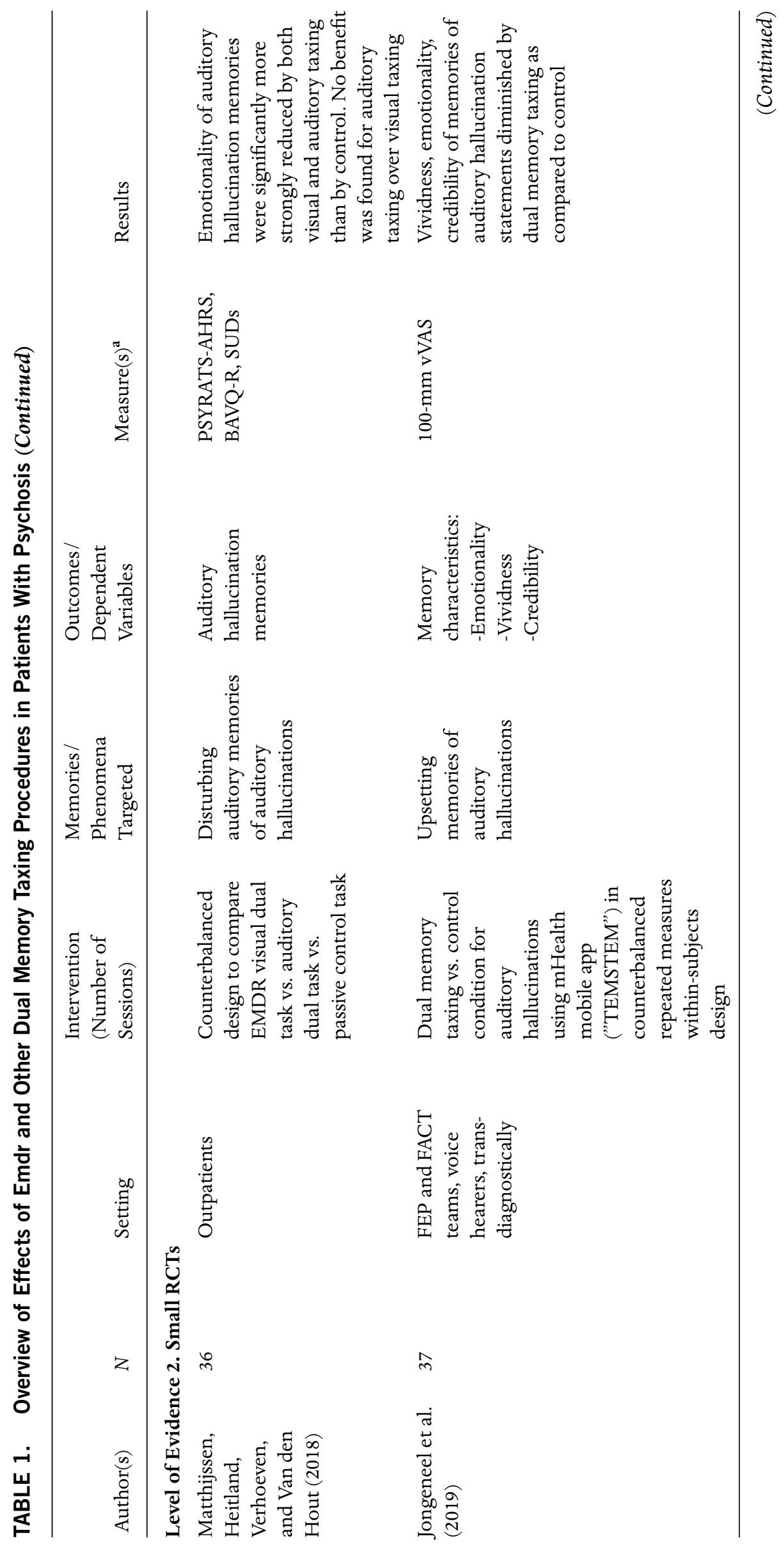




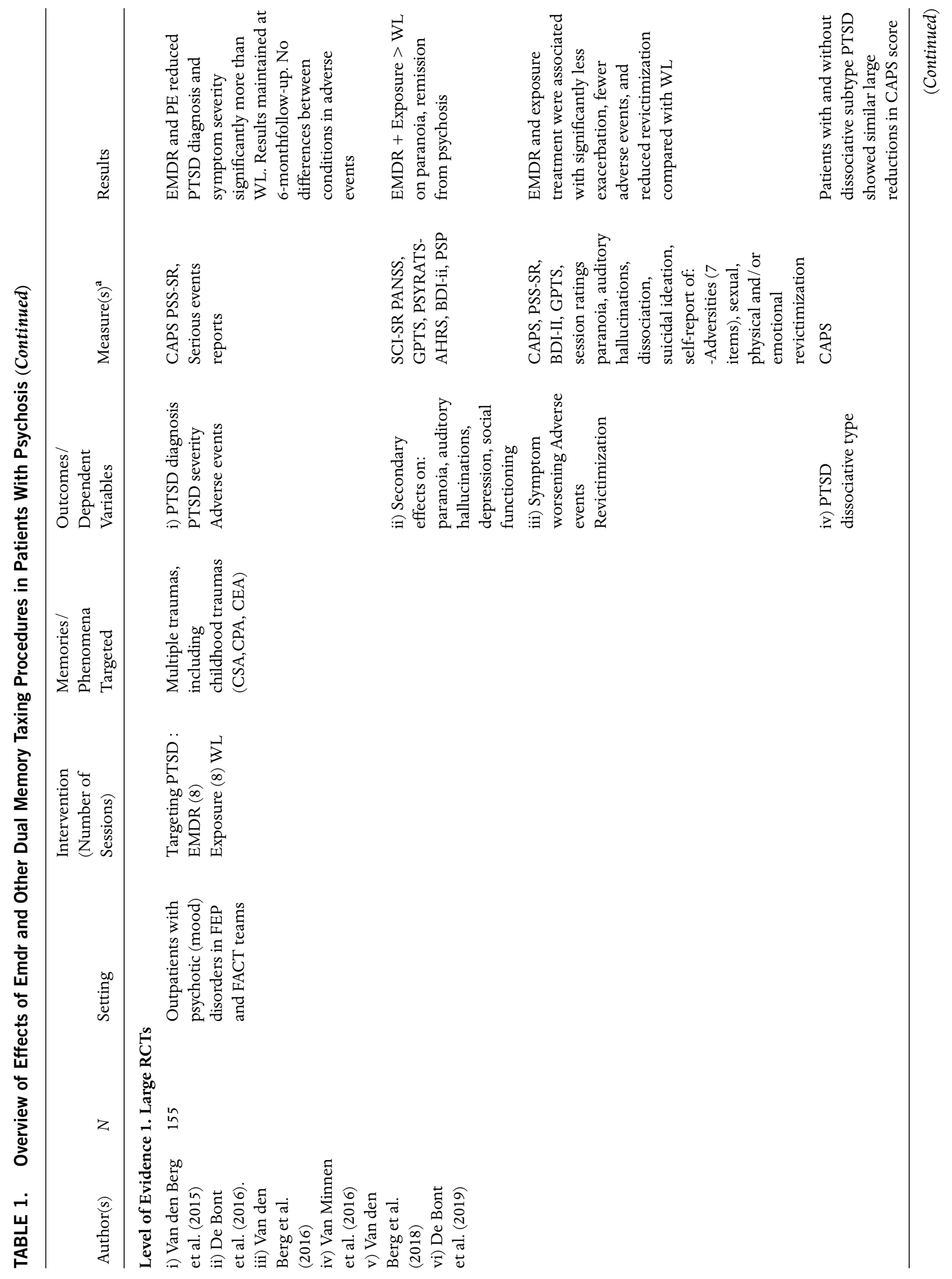




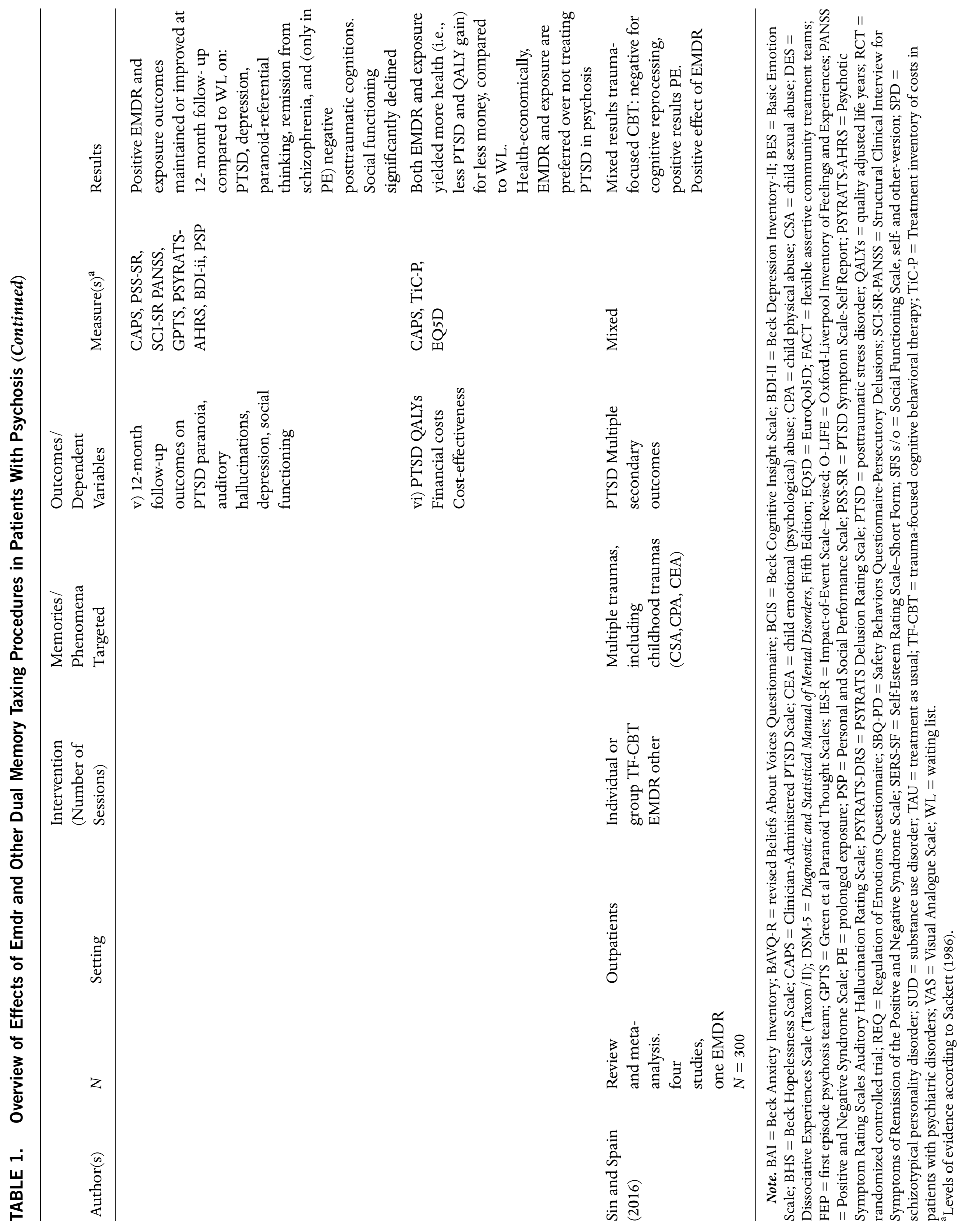


(TAU), the application of both PE and EMDR therapy was found to be associated with diminished adversities and symptom worsening in patients, while there were also indications that both therapies reduced the risk of revictimization (Van den Berg et al., 2016). As part of the research project the first cost-effectiveness analysis ever on EMDR was conducted, comparing the three conditions (TAU + EMDR vs. TAU + PE vs. TAU alone) from a health-economic perspective. In comparison to the no PTSD treatment condition (TAU alone), both treatment conditions were found to be associated with significantly more health gain (quality adjusted life years, QALY) and more remissions from PTSD for significantly lower total costs for society: $€ 1574$ (EMDR) and $-€ 422$ (PE) per patient in 6 months' time (De Bont et al., 2019). The findings from this research project indicate that there is no need for patients with psychotic disorders to be excluded from TFT.

Given that both EMDR and PE therapy have shown strong positive therapeutic effects in patients with PTSD among many different patient groups, experiments have now started to determine whether combining these therapies in intensive treatment programs are more effective than the usual monotherapeutic approaches (e.g., Van Woudenberg et al., 2018). In the future, research it would be interesting to test these intensive treatment programs in patients with psychotic disorders.

\section{EMDR for Psychotic Symptoms}

At the moment, for psychosis, the treatment options offered by CBT (including novel CBT approaches such as Virtual Reality-CBT) are by far the most extensively tested and empirically validated among the psychological therapies (e.g., see Pot-Kolder et al., 2018; van der Gaag, Valmaggia, \& Smit, 2014). However, bearing this in mind, EMDR therapy which is focussed on psychotic symptoms is a growing field of interest. For instance, in Korea, Kim et al. (2010) examined the application of EMDR in acutely admitted patients with schizophrenia but found no differences in effects between applying three sessions of EMDR therapy for adverse events (without reference to PTSD symptoms), TAU, and progressive muscle relaxation.

More recently, the application of specific traumasensitive psychosis protocols been proposed, fitting EMDR therapy procedures and conceptualizations with existing evidence-based cognitive behavioral models of psychosis (see Van den Berg, Van der Vleugel, Staring, De Bont, \& De Jongh, 2013; Van der
Vleugel, Van den Berg, De Bont, Staring, \& De Jongh, 2015). Also, a trauma-sensitive case conceptualization strategy, called ICONN, and case reviews of Miller (2016) are worth mentioning. However, the efficacy of such fairly complicated procedures involving EMDR, ego state therapy, and structural dissociation therapy for psychotic symptoms has not yet been tested in randomized controlled trials (RCTs; De Bont \& Geurink, 2016). Miller (2016) describes an approach in Japan (Kikuchi, 2008, published in Japanese only) applying EMDR therapy to schizophrenia in a context of psychoeducation and cognitive and cognitive behavioral therapy.

Because negative intrusive imagery plays an important role in psychosis (see e.g., Schulze, Freeman, Green, \& Kuipers, 2013, for more information), pilot case studies have started to examine the application of EMDR therapy procedures aimed at psychotic individuals' scary fantasies about the future, using Logie and De Jongh's (2014) flashforward technique. For example, Croes et al. (2014) investigated the effect of EMDR as a possible type of psychological treatment for patients suffering from psychosis-related imagery. The negative imagery of three psychotic outpatients who suffered from auditory hallucinations and delusions was treated with EMDR therapy in an average of six sessions. The treatment appeared to reduce patients' level of anxiety, depression, and the severity of psychotic symptoms. In addition, the patients reported less avoidant behavior and greater cognitive awareness. The results of this study suggest that EMDR can reduce vividness and emotionality of imagery in psychosis, thereby alleviating patients' psychotic symptoms. Obviously, these tentative findings require more rigorous tests using RCTs.

In addition to the negative imagery studies, other studies are now examining EMDR procedures for the reduction of memories with prominent auditory or visual features and of hallucinations. Important to note that intrusive memories and hallucinations may share a common ground in trauma in that it has been suggested that, in patients with psychotic disorders, some hallucinations may be conceptualized as decontextualized memory intrusions (Hardy, 2017; McCarthy-Jones \& Longden, 2015; Morrison, Frame, $\&$ Larkin, 2003).

Some researchers found that the dual tasking involved in EMDR therapy is associated with reduced memory emotionality and vividness of intrusive memories with strong auditory or visual features (Matthijssen, Van Schie, \& Van den Hout, 2018; Matthijssen, Verhoeven, Van den Hout, \& Heitland, 2017). They also examined whether taxing working 
memory (performing a dual task while recalling a memory) in a modality-specific way (auditory taxing for auditory memories and visual taxing for visual memories) might be even more effective in reducing vividness and emotionality of memories. To this end, two studies are noteworthy. While Matthijssen et al. $(2018,2019)$ examined the effects of different forms of memory taxation on auditory hallucination memories, Jongeneel et al. (2019) applied memory taxation targeting auditory hallucinations in a context of mHealth, using a mobile app (details of this trial are published in the study protocol, Jongeneel, Scheffers, $\&$ Tromp, 2018). The results of both studies suggest that vividness, emotionality, and in the latter also credibility of memories of auditory hallucination statements, could be diminished by dual memory taxing.

\section{Discussion}

In this contribution we presented a short overview of EMDR studies in the field of psychosis. It appears that TFT focused on PTSD or symptoms of psychosis, including EMDR therapy, can be beneficial for these patients, assuming the EMDR treatment is embedded in TAU for psychosis. Interesting and clinically relevant is that studies achieved positive results without using preparatory stabilization procedures and without major adjustments of the basic EMDR therapy procedures (see De Jongh et al., 2016 for a critical analysis of the issue of a stabilization phase prior to TFT). While acknowledging that TFT can be burdensome for patients, research is mounting suggesting that leaving trauma-related symptomatology in psychotic patients untreated is more disadvantageous than offering TFT, both clinically and economically.

In the NICE guidelines for psychosis (NICE, 2014) and PTSD (NICE, 2005), the option of treatment of comorbid PTSD is now included for service users suffering from psychosis. Therefore, we strongly support the implementation in clinical practice of trauma-focused instruments and interventions. Firstly, implementation of validated screening questionnaires (e.g., TSQ) and diagnostic instruments might help to remedy the grave underdetection of trauma and PTSD in patients with psychosis. Secondly, we recommend the training in-and application of-evidence-based TFT protocols such as EMDR therapy and/or CBT (PE or cognitive reprocessing $[\mathrm{CR}]$ ).

It is hoped that in a few years new data and knowledge involving the effects of evidence-based TFT protocols (i.e., EMDR, PE, and CR) for PTSD and trauma-related symptoms of psychosis in patients with psychotic disorders will become available. One initiative is currently being implemented in the Netherlands (the RE.PROCESS study), another in Spain (https: / / www.smartpatients .com/trials/NCT03991377). In the multicenter, single-blind RCT "RE.PROCESS," PTSD and traumarelated psychotic symptoms are subsequently targeted in four conditions: two directly TFT, EMDR, and PE therapy, are compared to the indirectly TFT of cognitive restructuring therapy, and a waiting list control condition. In the Spanish single-blind, multicenter RCT, the effectiveness of adding EMDR to TAU is compared to TAU alone in patients with first psychotic episodes and a history of trauma.

Closely related to the studies focusing on PTSD in psychosis is a newly emerged line of EMDR research that addresses psychotic symptomatology directly. The purpose of this research is to examine whether the application of EMDR therapy might influence hallucinations, delusional ideation, or negative intrusive imagery that plays an important role in the development and maintenance of psychotic symptoms. Strong evidence for these applications is currently lacking, but research efforts in this area are increasing. A pilot RCT examining EMDR for traumarelated psychotic symptomatology is under way in the United Kingdom (the EYES trial; http://www. isrctn.com/ISRCTN16262847), as is the aforementioned RE.PROCESS study on EMDR, PE, and CR therapies.

In conclusion, albeit research on EMDR therapy for psychosis is only at its initial stage, the first studies show promising and interesting outcomes in that adding TFT to the armamentarium used for patients with psychotic disorders might be beneficial for health and clinical gains as well as the economic costs. Therefore, among the psychological therapies, EMDR therapy deserves a place in psychosis care and research. A crucial precondition for actually bringing about beneficial effects is that mental healthcare services and researchers let go of their own hesitations and proceed with examining, disseminating, and implementing rationally, empirically validated TFT for patients with psychosis and trauma-related symptomatology.

\section{References}

Arseneault, L., Cannon, M., Fisher, H. L., Polanczyk, G., Moffitt, T. E., \& Caspi, A. (2011). Childhood trauma and children's emerging psychotic symptoms: A genetically sensitive longitudinal cohort study. The American Journal of Psychiatry, 168(1), 65-72. doi:10.1176/appi.ajp.2010.10040567 
Bendall, S., Alvarez-Jimenez, M., Hulbert, C. A., McGorry, P. D., \& Jackson, H. J. (2012). Childhood trauma increases the risk of post-traumatic stress disorder in response to first-episode psychosis. Australian and New Zealand Journal of Psychiatry, 46(1), 35-39. doi: $10.1177 / 0004867411430877$

Bentall, R. P., Wickham, S., Shevlin, M., \& Varese, F. (2012). Do specific early-life adversities lead to specific symptoms of psychosis? A study from the 2007 the Adult Psychiatric Morbidity Survey. Schizophrenia Bulletin, 38(4), 734-740. doi:10.1093/schbul/sbs049

Björkenstam, E., Burström, B., Vinnerljung, B., \& Kosidou, K. (2016). Childhood adversity and psychiatric disorder in young adulthood: An analysis of 107,704 Swedes. Journal of Psychiatric Research, 77, 67-75. doi:10.1016/j.jpsychires.2016.02.018

Croes, C. F., Van Grunsven, R., Staring, A. B. P., Van den Berg, D. P. G., De Jongh, A., \& Van der Gaag, M. (2014). Mentale beelden bij psychose: EMDR als een nieuwe interventie bij het behandelen van stemmen en wanen [Imagery in psychosis: EMDR as a new intervention in the treatment of delusions and auditory hallucinations]. Tijdschrift voor Psychiatrie, 56(9), 568-576.

De Bont, P., Van den Berg, D. P., Van der Vleugel, B. M., De Roos, C., De Jongh, A., Van der Gaag, M., \& Van Minnen, A. (2016). Prolonged exposure and EMDR for PTSD v. a PTSD waiting-list condition: Effects on symptoms of psychosis, depression and social functioning in patients with chronic psychotic disorders. Psychological Medicine, 46, 2411-2421. doi:10.1017/S0033 291716001094

De Bont, P., Van Minnen, A., \& De Jongh, A. (2013). Treating ptsd in patients with psychosis: A within-group controlled feasibility study examining the efficacy and safety of evidence-based PE and EMDR protocols. Behavior Therapy, 44(4), 717-730. doi:10.1016/j.beth.2013.07.002

De Bont, P. A., \& Geurink, M. (2016). A review of psychosis and trauma: EMDR for trauma related problems in patients with psychosis by Paul William Miller. PsycCRITIQUES, 16(14).

De Bont, P. A. J. M., Van den Berg, D. P. G., Van der Vleugel, B. M., De Roos, C., De Jongh, A., Van der Gaag, M., \& Van Minnen, A. (2015). Predictive validity of the trauma screening questionnaire in detecting post-traumatic stress disorder in patients with psychotic disorders. British Journal of Psychiatry, 408-416. doi:10.1192/bjp.bp.114.148486

De Bont, P. A. J. M., Van den Berg, D. P. G., Van der Vleugel, B. M., De Roos, C., Mulder, C., Becker, E. S., . . . Van Minnen, A. (2013). A multi-site single blind clinical study to compare the effects of prolonged exposure, eye movement desensitization and reprocessing and waiting list on patients with a current diagnosis of psychosis and co morbid post traumatic stress disorder: Study protocol for the randomized controlled trial treating trauma in psychosis. Trials, 14(1), 151. doi:10.1186/1745-621514-151
De Bont, P., Van den Berg, D., Van der Vleugel, B., De Roos, C., De Jongh, A., Van der Gaag, M., \& Van Minnen, A. (2018). Long-term outcomes of traumafocused treatment in psychosis. The British Journal of Psychiatry, 212, 1-3. doi:10.1192/bjp.2017.30

De Bont, P. A. J. M., Van der Vleugel, B. M., Van den Berg, D. P. G., De Roos, C., Lokkerbol, J., Smit, F., . . V Van Minnen, A. (2019). Health-economic benefits of treating trauma in psychosis. European Journal of Psychotraumatology, 10(1), 1565032-1565032. doi:10.1080/20008198.2018 .1565032

De Jongh, A., Resick, P. A., Zoellner, L. A., Van Minnen, A., Lee, C. W., Monson, C. M., ... Rauch, S. A. (2016). Critical analysis of the current treatment guidelines for complex PTSD in adults. Depression and Anxiety, 33(5), 359-369. doi:10.1002/da.22469

Frueh, B. C., Grubaugh, A. L., Cusack, K. J., Kimble, M. O., Elhai, J. D., \& Knapp, R. G. (2009). Exposurebased cognitive-behavioral treatment of PTSD in adults with schizophrenia or schizoaffective disorder: A pilot study. Journal of Anxiety Disorders, 23(5), 665-675. doi:10.1016/j.janxdis.2009.02.005

Hardy, A. (2017). Pathways from trauma to psychotic experiences: A theoretically informed model of posttraumatic stress in psychosis. Frontiers in Psychology, 8, 697.

Hassan, A. N., \& De Luca, V. (2015). The effect of lifetime adversities on resistance to antipsychotic treatment in schizophrenia patients. Schizophrenia Research, 161(2), 496-500. doi:10.1016/j.schres.2014.10.048

Jongeneel, A., Scheffers, D., Tromp, N., Nuij, C., Delespaul, P., Riper, H., . . . Van den Berg, D. (2018). Reducing distress and improving social functioning in daily life in people with auditory verbal hallucinations: study protocol for the 'Temstem' randomised controlled trial. BMJ Open, 8(3), [e020537]. https: / / doi.org/10.1136/bmjopen-2017-020537

Jongeneel, A., van Veen, S. C., Scheffers, D., Riper, H., Van den Hout, M. A., Van der Gaag, M., \& Van den Berg, D. (2019). Dual tasking reduces salience and credibility of voice memories in voice-hearing individuals: Results from a controlled trial. Manuscript submitted for publication.

Kikuchi, A. (2008). Application of EMDR for schizophrenia. Clinical Psychology: Various Aspects, 27(2), 317-324.

Kim, D., Choi, J., Kim, S. H., Oh, D. H., Park, S. C., \& Lee, S. H. (2010). A pilot study of brief eye movement desensitization and reprocessing (EMDR) for treatment of acute phase schizophrenia. Korean Journal of Biological Psychiatry, 17(2), 94-102.

Kratzer, L., Heinz, P., \& Schennach, R. (2017). Significant improvement of posttraumatic stress disorder and psychotic symptoms after inpatient eye movement desensitization and reprocessing treatment: A case report with 6-month follow-up. Indian journal of psychiatry, 59(3), 389-390. doi:10.4103/ psychiatry.IndianJPsychiatry_318_16

Kuijpers, K. F., Van der Knaap, L. M., \& Winkel, F. W. (2012). PTSD symptoms as risk factors for intimate 
partner violence revictimization and the mediating role of victims' violent behavior. Journal of Traumatic Stress, 25(2), 179-186. doi:10.1002/jts.21676

Laugharne, R., Marshall, D., Laugharne, J., \& Hassard, A. (2014). A role for EMDR in the treatment of trauma in patients suffering from a psychosis: Four vignettes. Journal of EMDR Practice and Research, 8(1), 19-24. doi:10.1891/1933-3196.8.1.19

Lecomte, T., Spidel, A., Leclerc, C., MacEwan, G. W., Greaves, C., \& Bentall, R. P. (2008). Predictors and profiles of treatment non-adherence and engagement in services problems in early psychosis. Schizophrenia Research, 102(1), 295-302. doi:10.1016/j.schres.2008.01.024

Linscott, R., \& Van Os, J. (2013). An updated and conservative systematic review and meta-analysis of epidemiological evidence on psychotic experiences in children and adults: On the pathway from proneness to persistence to dimensional expression across mental disorders. Psychological Medicine, 43(6), 1133-1149. doi:10.1017/S0033291712001626

Logie, R., \& De Jongh, A. (2014). The 'Flashforward procedure': Confronting the catastrophe. Journal of EMDR Practice and Research, 8, 25-32. doi:10.1891/19333196.8.1.25

Lommen, M. J. J., \& Restifo, K. (2009). Trauma and posttraumatic stress disorder (PTSD) in patients with schizophrenia or schizoaffective disorder. Community Mental Health Journal, 45(6), 485-496. doi:10.1007/ s10597-009-9248-x

Lysaker, P., and LaRocco, V. (2008). The prevalence and correlates of trauma-related symptoms in schizophrenia spectrum disorder. Comprehensive Psychiatry, 49(4), 330-334. doi:10.1016/j.comppsych.2007.12.003

Lysaker, P. H. P., Buck, K. D. M. A. B., \& LaRocco, V. A. M. (2007). Clinical and psychosocial significance of trauma history: In the treatment of schizophrenia. Journal of Psychosocial Nursing \& Mental Health Services, 45(8), 44-51.

Maniglio, R. (2009). Severe mental illness and criminal victimization: A systematic review. Acta Psychiatrica Scandinavica, 119, 180-191. doi:10.1111/j.16000447.2008.01300.x

Matheson, S. L., Shepherd, A. M., Pinchbeck, R. M., Laurens, K. R., \& Carr, V. J. (2013). Childhood adversity in schizophrenia: A systematic meta-analysis. Psychological Medicine, 43(2), 225-238. doi:10.1017/ S0033291712000785

Matthijssen, S. J. M. A., Heitland, I., Verhoeven, L. C. M., \& van den Hout, M. A. (2019). Reducing the emotionality of auditory hallucination memories in patients suffering from auditory hallucinations. Frontiers in Psychiatry, 10. doi:10.3389/fpsyt.2019.00637

Matthijssen, S., van Schie, K., \& Hout, M. (2018). The Effect of modality specific interference on working memory in recalling aversive auditory and visual memories. Cognition \& Emotion, 33, 1-12. doi:10.1080/02699931.2018.1547271
Matthijssen, S. J. M. A., Verhoeven, L. C. M., Van den Hout, M. A., \& Heitland, I. (2017). Auditory and visual memories in PTSD patients targeted with eye movements and counting: The effect of modality-specific loading of working memory. Frontiers in Psychology, 8, 1937. doi:10.3389/fpsyg.2017.01937

McCarthy-Jones, S., \& Longden, E. (2015). Auditory verbal hallucinations in schizophrenia and post-traumatic stress disorder: common phenomenology, common cause, common interventions? Frontiers in Psychology, 6(1071). Retrieved from https://www.frontiersin.org/ article/10.3389/fpsyg.2015.01071

Meyer, J. M., Farrell, N. R., Kemp, J. J., Blakey, S. M., \& Deacon, B. J. (2014). Why do clinicians exclude anxious clients from exposure therapy? Behaviour Research and Therapy, 54, 49-53. doi:10.1016/j.brat.2014.01.004

Miller, P. W. (2016). EMDR therapy for schizophrenia and other psychoses. New York, NY: Springer Publishing.

Morrison, A. P., Frame, L., \& Larkin, W. (2003). Relationships between trauma and psychosis: A review and integration. British Journal of Clinical Psychology, 42(4), 331-353. doi:10.1348/014466503322528892

Mueser, K. T., Gottlieb, J. D., Xie, H., Lu, W., Yanos, P. T., Rosenberg, S. D., . . McHugo, G. J. (2015). Evaluation of cognitive restructuring for post-traumatic stress disorder in people with severe mental illness. The British Journal of Psychiatry: The Journal of Mental Science, 206(6), 501-508. doi:10.1192/bjp.bp.114.147926

Mueser, K. T., Lu, W., Rosenberg, S. D., \& Wolfe, R. (2010). The trauma of psychosis: Posttraumatic stress disorder and recent onset psychosis. Schizophrenia Research, 116(2-3), 217-227. doi:10.1016/j.schres.2009.10.025

Mueser, K. T., Rosenberg, S. D., Xie, H., Jankowski, M. K., Bolton, E. E., Lu, W., . . W Wolfe, R. (2008). A randomized controlled trial of cognitive-behavioral treatment for posttraumatic stress disorder in severe mental illness. Journal of Consulting and Clinical Psychology, 76(2), 259-271. doi:10.1037/0022-006x.76.2.259

National Institute for Health and Care Excellence. (2014). Psychosis and schizophrenia in adults: Key priorities for implementation. Retrieved from https://www.nice. org.uk/guidance/cg178/ chapter/Key-priorities-forimplementation

National Institute for Clinical Excellence. (2005). Posttraumatic stress disorder: The management of PTSD in adults and children in primary and secondary care. Retrieved from https: / / www.ncbi.nlm.nih.gov/books/NBK56494/.

Pot-Kolder, R. M. C. A., Geraets, C. N. W., Veling, W., van Beilen, M., Staring, A. B. P., Gijsman, H. J., . . . van der Gaag, M. (2018). Virtual-reality-based cognitive behavioural therapy versus waiting list control for paranoid ideation and social avoidance in patients with psychotic disorders: A single-blind randomised controlled trial. The Lancet Psychiatry, 5(3), 217-226. doi:10.1016/S2215-0366(18)30053-1

Rodrigues, R., \& Anderson, K. K. (2017). The traumatic experience of first-episode psychosis: A systematic 
review and meta-analysis. Schizophrenia Research, 189(Suppl. C), 27-36. doi:10.1016/j.schres.2017.01.045

Ronconi, J. M., Shiner, B., \& Watts, B. V. (2014). Inclusion and exclusion criteria in randomized controlled trials of psychotherapy for PTSD. Journal of Psychiatric Practice, 20(1), 25-37. doi:10.1097/01.pra.0000442936.23457.5b

Sackett, D. L. (1986). Rules of evidence and clinical recommendations on the use of antithrombotic agents. Chest, 89(Suppl. 2), 2S-3S.

Sautter, F. J., Brailey, K., Uddo, M. M., Hamilton, M. F., Beard, M. G., \& Borges, A. H. (1999). PTSD and comorbid psychotic disorder: Comparison with veterans diagnosed with PTSD or psychotic disorder. Journal of Traumatic Stress, 12(1), 73-88. doi:10.1023/ A:1024794232175

Schulze, K., Freeman, D., Green, C., \& Kuipers, E. (2013). Intrusive mental imagery in patients with persecutory delusions. Behaviour Research and Therapy, 51(1), 7-14. doi:10.1016/j.brat.2012.10.002

Seow, L. S. E., Ong, C., Mahesh, M. V., Sagayadevan, V., Shafie, S., Chong, S. A., \& Subramaniam, M. (2016). A systematic review on comorbid post-traumatic stress disorder in schizophrenia. Schizophrenia Research, 176(2), 441-451. doi:10.1016/j.schres.2016.05.004

Shevlin, M., Houston, J. E., Dorahy, M. J., \& Adamson, G. (2007). Cumulative traumas and psychosis: An analysis of the national comorbidity survey and the british psychiatric morbidity survey. Schizophrenia Bulletin, 34(1), 193-199. doi:10.1093/schbul/sbm069

Sin, J., \& Spain, D. (2016). Psychological interventions for trauma in individuals who have psychosis: A systematic review and meta-analysis. Psychosis, 9(1), 67-81. doi:10.1080/17522439.2016.1167946

Trotta, A., Murray, R., \& Fisher, H. (2015). The impact of childhood adversity on the persistence of psychotic symptoms: A systematic review and metaanalysis. Psychological Medicine, 45(12), 2481-2498. doi:10.1017/S0033291715000574

Van den Berg, D. P., De Bont, P. A., Van der Vleugel, B. M., De Roos, C., De Jongh, A., Van Minnen, A., \& Van der Gaag, M. (2015). Prolonged exposure vs eye movement desensitization and reprocessing vs waiting list for posttraumatic stress disorder in patients with a psychotic disorder: A randomized clinical trial. JAMA Psychiatry, 72(3), 259-267. doi:10.1001/jamapsychiatry.2014.2637

Van den Berg, D. P. G., De Bont, P. A. J. M., Van der Vleugel, B. M., De Roos, C., De Jongh, A., Van Minnen, A., \& Van der Gaag, M. (2016). Trauma-focused treatment in PTSD patients with psychosis: Symptom exacerbation, adverse events, and revictimization. Schizophrenia Bulletin, 42(3), 693-702. doi:10.1093/schbul/sbv172

Van den Berg, D. P. G., \& Van der Gaag, M. (2012). Treating trauma in psychosis with EMDR: A pilot study. Journal of Behavior Therapy and Experimental Psychiatry, 43(1), 664-671. doi:10.1016/j.jbtep.2011.09.011

Van den Berg, D. P., Van der Vleugel, B. M., Staring, A. B., De Bont, P. A., \& De Jongh, A. (2013). EMDR in psychosis: Guidelines for conceptualization and treatment. Journal of EMDR Practice and Research, 7(4), 208-224. doi:10.1891/1933-3196.7.4.208

Van der Gaag, M., Valmaggia, L. R., \& Smit, F. (2014). The effects of individually tailored formulation-based cognitive behavioural therapy in auditory hallucinations and delusions: A meta-analysis. Schizophrenia Research, 156(1), 30-37. doi:10.1016/j.schres.2014.03.016

Van der Vleugel, B., Van den Berg, D., De Bont, D., Staring, T., \& De Jongh, A. (2015). EMDR Therapy for traumatized patients with psychosis. In M. Luber (Ed.), Eye movement desensitization and reprocessing: EMDR scripted protocols and summary sheets. Treating trauma-and stressorrelated conditions (pp. 97-148). New York, NY: Springer Publishing.

Van Minnen, A., Van der Vleugel, B. M., Van den Berg, D. P., De Bont, P., De Roos, C., Van der Gaag, M., \& De Jongh, A. (2016). Effectiveness of trauma-focused treatment for patients with psychosis with and without the dissociative subtype of post-traumatic stress disorder. The British Journal of Psychiatry, 209(4), 347-348. doi:10.1192/bjp.bp.116.185579

Van Woudenberg, C., Voorendonk, E. M., Bongaerts, H., Zoet, H. A., Verhagen, M., Lee, C. W., . . . De Jongh, A. (2018). Effectiveness of an intensive treatment programme combining prolonged exposure and eye movement desensitization and reprocessing for severe post-traumatic stress disorder. European Journal of Psychotraumatology, 9(1), 1487225. doi:10.1080/20008198.2018.1487225

Varese, F., Smeets, F., Drukker, M., Lieverse, R., Lataster, T., Viechtbauer, W., . . Bentall, R. P. (2012). Childhood adversities increase the risk of psychosis: a meta-analysis of patient-control, prospective- and cross-sectional cohort studies. Schizophrenia Bulletin, 38(4), 661-671 doi:10.1093/schbul/sbs050

Ward-Brown, J., Keane, D., Bhutani, G., Malkin, D., Sellwood, B., \& Varese, F. (2018). TF-CBT and EMDR for young people with trauma and first episode psychosis (using a phasic treatment approach): Two early intervention service case studies. The Cognitive Behaviour Therapist, 11, E17. doi:10.1017/S1754470X18000193

Disclosure. David van den Berg receives income for published books on psychotic disorders and for the training of postdoctoral professionals in the treatment of psychotic disorders. Ad de Jongh receives income from published books on EMDR therapy and for the training of postdoctoral professionals in this approach. Paul de Bont receives income for the training of postdoctoral professionals in this approach.

Correspondence regarding this article should be directed to Paul de Bont, PhD, Clinical Psychologist, Mental Health Service GGZ Oost Brabant, Bilderbeekstraat 44, 5831 CX Boxmeer, the Netherlands. E-mail: paj.de.bont@ggzoostbrabant.nl 